\title{
Monitoramento do sedimento suspenso no Lago Catalão (Iranduba, AM) com dados Landsat-8
}

\author{
Rogério Ribeiro Marinho, Flávio Wachholz, Renata Souza
}

https://doi.org/10.4322/mp.978-65-991393-8-3.c3

\begin{abstract}
Resumo
A região de confluência entre o rio Negro e Solimões é uma extensa área de mistura de águas que possuem diferenças significativas de suas características físicas e químicas, principalmente quanto ao transporte de sedimentos suspensos. A dinâmica fluvial nesta planície de inundação é controlada pelo rio Solimões, que ocasiona significativas mudanças na paisagem, principalmente pela entrada de sedimentos suspensos em lagos conectados ao sistema fluvial. Este trabalho teve como objetivo analisar a variação espacial e temporal do sedimento suspenso no Lago Catalão (ilha da Xiborena). Dados de reflectância da banda do vermelho do sensor OLI, satélite Landsat-8, foram utilizados para estimar a concentração de sedimento suspenso (CSS). A variabilidade anual da CSS nesta área de estudo é controlada principalmente pelo regime hidrológico dos rios Solimões e Negro. Estimativas da CSS com imagens do Landsat-8 indica uma concentração média anual de $12,04 \mathrm{mg} \mathrm{L}^{-1} \pm 35 \%$, com menor concentração no período de enchente $\left(8,20 \mathrm{mg} \mathrm{L}^{-1}\right)$ e maior durante a cheia $(15,09 \mathrm{mg}$ $\mathrm{L}^{-1}$ ). Entre os meses de agosto a novembro (vazante e seca) ocorre a menor disponibilidade de imagens para o período analisado (2013-2020) devido à cobertura de nuvens na região e a resolução temporal do sensor OLI (16 dias). Por fim, este trabalho realiza uma discussão a respeito do uso de sensores ópticos para o monitoramento da paisagem fluvial da região de confluência dos rios Negro e Solimões.
\end{abstract}

Palavras-chave: bacia amazônica, comportamento espectral da água, planície de inundação, transporte de sedimento suspenso.

\section{Introdução}

A bacia amazônica possui uma densa rede de drenagem e uma vasta planície de inundação, com complexos hidrossistemas que desempenham um importante papel na regulagem do ciclo hidrológico, em processos biogeoquímicos e na manutenção de paisagens. Estas planícies possuem sua dinâmica de inundação controlada pelo pulso de inundação [1], cuja variabilidade natural do nível d'água pode chegar a 18 metros nos rios da região $[2,3]$.

Atualmente o monitoramento hidrológico e sedimentológico nos principais hidrossistemas na Amazônia são realizados por meio de observações pontuais em estações hidrométricas, localizadas principalmente nos grandes rios. Nos últimos anos dados coletados por plataformas orbitais de sensoriamento remoto contribuíram significativamente para ampliar a aquisição de dados em diferentes ambientes 
brasileiros pobremente monitorados, possibilitando com isso análises detalhadas de lagos localizados na planície de inundação do rio Amazonas [4].

Embora exista uma considerável quantidade de estudos sobre a geomorfologia e a paisagem fluvial na bacia Amazônica, pesquisas realizadas nos últimos anos não tiveram como foco os lagos na região de confluência dos rios Negro e Solimões, uma extensa área com mistura de águas de dois grandes rios, que possuem diferenças significativas das características hidráulicas [5-7], na qualidade da água [8,9] e de grande importância para a população ribeirinha $[10,11]$. O rio Negro, com baixo pH e águas escuras, drenam principalmente terrenos antigos dos escudos das Guianas, enquanto que o rio Solimões, com águas brancas, transporta sedimentos suspensos oriundos da cordilheira dos Andes, com concentrações cerca de 30 vezes maior que 0 rio Negro [12]. Desta forma, as águas do Lago Catalão correspondem a uma mistura destes dois grandes rios fisicamente e quimicamente distintos [13].

O estudo de sedimentos suspensos na bacia amazônica é essencial para o entendimento da paisagem fluvial e sua relação com a gênese, morfodinâmica e ciclagem biogeoquímica de sistemas lacustres sob influência de grandes rios, bem como, para avaliação e mitigação de impactos de eventos climáticos extremos ou oriundos de atividades antrópicas que afetam a qualidade e disponibilidade de água [14]. Assim, análises geográficas deste tipo de paisagem em suas componentes espaciais e temporais, com uso de dados de campo e de sensoriamento remoto contribuem para o entendimento do intercâmbio de águas nestes hidrossistemas e como a população ribeirinha produz seu espaço geográfico. Além do mais, dados de sensoriamento remoto podem fornecer uma visão sinótica, aquisição em diferentes períodos do ano para subsidiar análise do funcionamento e transformações que um sistema aquático está sujeito [15].

Apesar da maior disponibilidade de dados de sensoriamento remoto, poucos estudos sobre o transporte de sedimento suspenso foram desenvolvidos em lagos sob influência dos rios Negro e Solimões, situação que contribui no baixo conhecimento da variabilidade espaço-temporal da qualidade da água nesta paisagem. Neste contexto, este trabalho tem como objetivo analisar a variação espaço-temporal do sedimento suspenso no Lago Catalão (ilha da Xiborena), região da confluência dos rios Negro e Solimões, por meio de dados dos sensores OLI (Landsat-8).

Os resultados apresentados neste trabalho buscam contribuir para a caracterização da dinâmica da paisagem em um lago com grande importância socioambiental da região do encontro das águas, visto a dependência da população local de atividades de pesca e turismo [11], piscicultura [16] e pelo fato deste lago ser considerado um importante berçário para espécies de peixes oriundos do rio Solimões $[17,18]$.

\section{Material e Métodos}

\section{1. Área de estudo}

O Lago Catalão localiza-se na ilha Xiborena, que por sua vez está localizada na margem direita do rio Negro e é parcialmente conectado ao rio Solimões (Figura 1). Esta área pertence ao município de Iranduba, porém seu acesso é realizado principalmente pelo Porto da Ceasa, em Manaus, estado do Amazonas. Na localidade denominada "Boca do Catalão" existe um assentamento de 110 casas flutuantes e uma população estimada de 350 habitantes [16] em 90 famílias distribuídas ao longo de canais (localmente conhecido como paranás) que conectam o lago ao rio Negro [11]. 


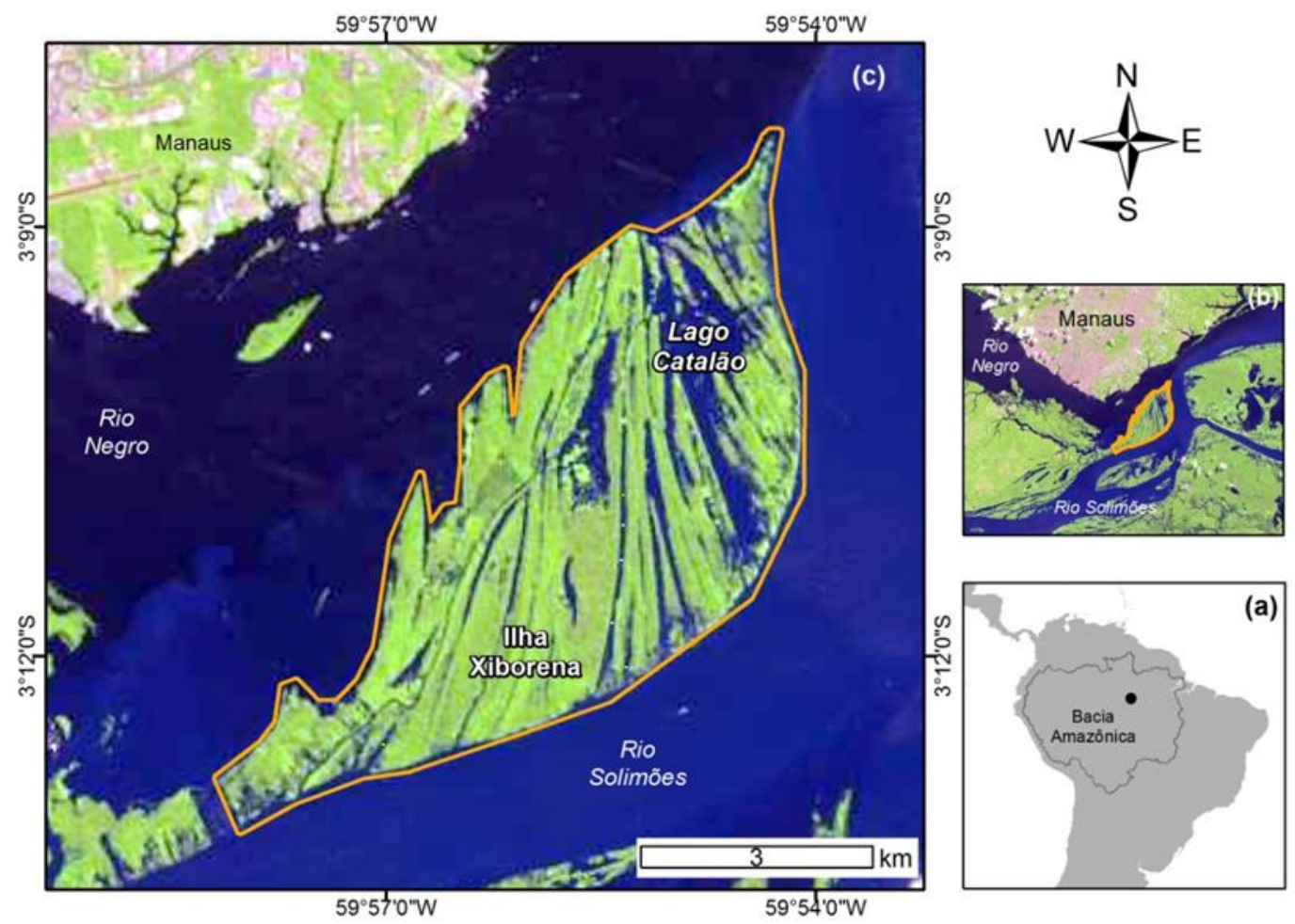

Figura 1. Localização da área de estudo. (a) Bacia Amazônica. (b) Confluência dos rios Negro e Solimões. (c) Lago Catalão (ilha Xiborena). Imagem RGB (6-5-4) do sensor OLI/Landsat-8 adquirida em 27/05/2017.

Este lago recebe águas de ambos os rios Negro e Solimões e é considerado um lago de águas mistas, ou seja, brancas e pretas [19]. A área do Lago Catalão aumenta e diminui de tamanho de acordo com o nível dos rios adjacentes, conectando-se normalmente ao rio Solimões através de um curto canal, após os primeiros meses de enchente e raramente perde sua conexão com o rio Negro. Em períodos de secas intensas, o lago se resume em uma pequena área denominada "poção" permanecendo conectado com o rio Negro [17].

A principal classificação empregada para descrever os tipos de água na Amazônia foi proposta por Harald Sioli na década de 1950 [20] com base nas propriedades ópticas aparentes [21] e suas relações com a biogeoquímica. As águas brancas possuem alta quantidade de sedimento inorgânico suspenso e são oriundas principalmente dos Andes, enquanto que as águas pretas possuem como principal característica a baixa concentração de sedimento inorgânico suspenso e elevada carga de matéria orgânica dissolvida. Já as águas claras apresentam maior transparência em relação às anteriores, porém com maior produtividade primária do fitoplâncton [22].

A Figura 2 apresenta espectros representativos da reflectância de sensoriamento remoto da água na área de estudo, onde se destaca as variações na forma e magnitude dos espectros coletados no Lago Catalão (Espectro 2) e na área sobre influência dos rios Negro (Espectro 3) e Solimões (Espectro 1). De forma geral, o sedimento suspenso do rio Solimões ocasiona maior energia refletida em relação aos espectros da reflectância da água sob influência do rio Negro. Devido à presença da matéria orgânica dissolvida na água do rio Negro os lagos e canais nesta região apresentam baixa energia refletida. Porém, destaca-se que em ambos os espectros 0 pico de reflectância ocorre na região espectral do vermelho $(690 \mathrm{~nm})$. 

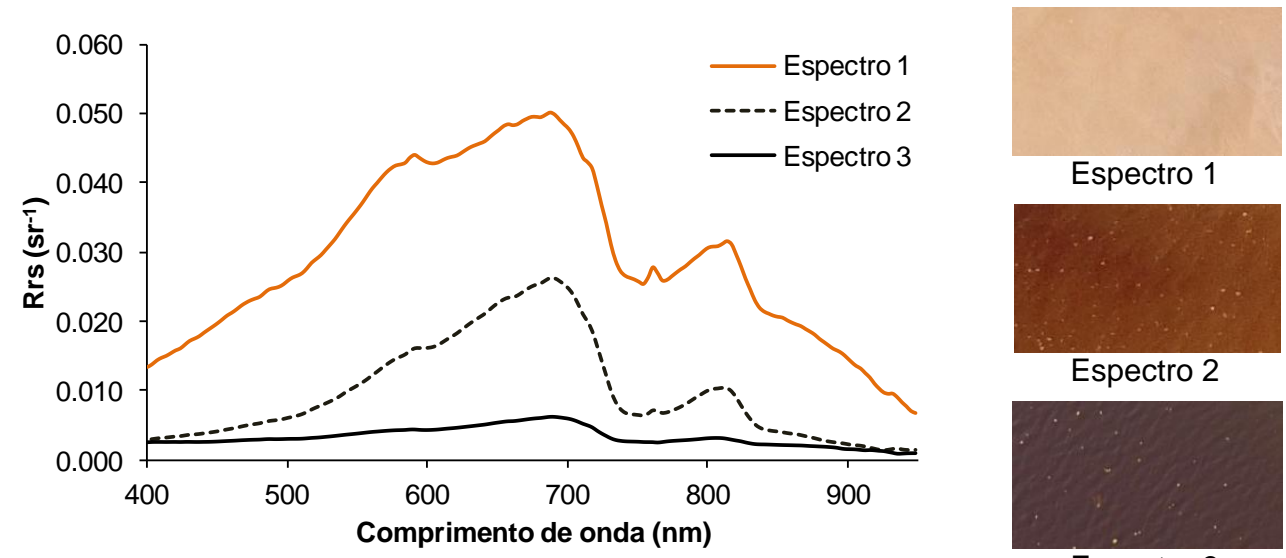

Espectro 3

Figura 2. Comportamento espectral da reflectância de sensoriamento remoto $\left(R_{r s}\right)$ da superfície d'água na região do Lago Catalão. Dados de abril de 2018.

O Lago Catalão possui área de aproximadamente $1,54 \mathrm{~km}^{2}$, apresenta formato alongado (largura máxima de $1,5 \mathrm{~km}$ e comprimento máximo de $4,5 \mathrm{~km}$ ) e um tempo médio de residência da água estimado em 148 dias [23]. Segundo Brito et al. [13] a água neste lago possui pouca oxigenação $\left(2,4 \mathrm{mg} \mathrm{L}^{-1}\right)$, baixo $\mathrm{pH}$ (variando de 6 a 7) e condutividade elétrica variando de 22 a $82\left(\mu \mathrm{cm}^{-1}\right)$, com as características físicas e químicas da água sob maior influência do rio Solimões durante o período de cheia.

A precipitação média anual na área de estudo é da ordem de $2280 \mathrm{~mm}$ por ano, com o período chuvoso ocorrendo entre dezembro e abril e o mês menos chuvoso em agosto. O regime hidrológico anual apresenta um comportamento regular, com cheia em junho-julho, vazante de agosto a setembro, seca entre outubro-novembro e período de enchente de dezembro a maio, apresentando variabilidade média anual do nível d'água de aproximadamente 10 metros [3].

\subsection{Material e Métodos}

A Figura 3 apresenta o fluxograma metodológico deste estudo. Foram utilizados dados de reflectância de sensoriamento remoto $\left(R_{r s}\right)$ e da concentração de sedimentos suspensos (CSS) para estimar sua dinâmica temporal e espacial no Lago Catalão através de imagens do sensor OLI (satélite Landsat-8). Os dados de $R_{r s}$ e CSS foram coletados em lagos e canais do baixo rio Negro e na região de confluência com o rio Solimões entre 2016 e 2020 em diferentes períodos hidrológicos.

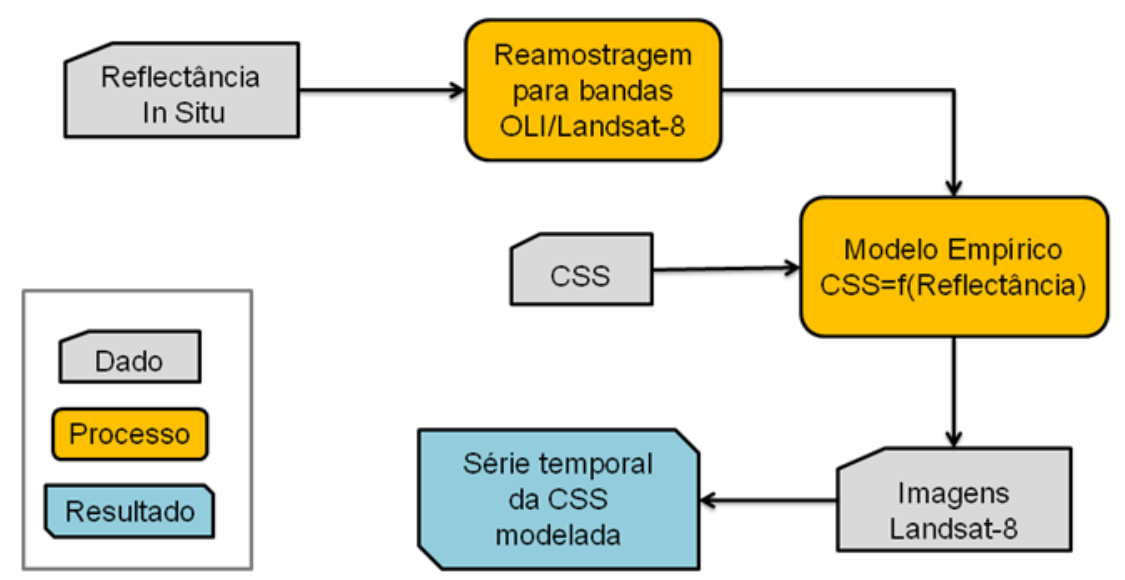

Figura 3. Fluxograma das etapas de processamento e análise de dados de sensoriamento remoto para estimar a concentração do sedimento suspenso. 
A reflectância de sensoriamento remoto $\left(R_{\mathrm{rs}}\right)$ foi obtida in situ com o uso de três radiômetros TriOs/RAMSES que operam no intervalo de 350-950 nm do espectro eletromagnético. A configuração geométrica para coleta dos dados radiométricos seguiu o protocolo de Mobley [24], com ângulo de visada de $40^{\circ}$ e azimute de $135^{\circ} \mathrm{em}$ relação ao Sol. $A R_{r s}$ para cada comprimento de onda $(\lambda)$ foi determinada a partir da equação 1:

$$
\mathrm{R}_{\mathrm{rs}}(\lambda)=\frac{\mathrm{L}_{\mathrm{t}}(\lambda)-\rho \cdot \mathrm{L}_{\mathrm{sky}}(\lambda)}{\mathrm{E}_{\mathrm{s}}(\lambda)}
$$

onde $E_{s}$ é a irradiância incidente na superfície da água em $90^{\circ}$, $L t$ é a radiância ascendente da superfície da água em $40^{\circ}$ off-nadir, $L_{s k y}$ é a radiância proveniente do céu em $40^{\circ}, \rho$ é um fator multiplicativo tabelado.

Para simular a reflectância medida pelos sistemas orbitais os espectros coletados in situ foram reamostrados para bandas do OLI/Landsat-8 utilizando a função de resposta espectral relativa deste sensor. A concentração de sedimentos suspensos (CSS, em $\mathrm{mg} \mathrm{L}^{-1}$ ) foi determinada seguindo o protocolo do Observatório HYBAM [25] na qual as amostras de água coletadas em superfície foram filtradas em membranas de acetato de celulose de $0,45 \mu \mathrm{m}$ de porosidade e pesadas em balanças analíticas.

Este estudo utilizou a modelagem empírica baseada na análise de regressão para estimar a CSS em função da $R_{r s}$. Esta abordagem foi utilizada pelo fato que a área de estudo possui valores da concentração de sedimentos suspensos que variam de baixa a moderada, ou seja, CSS menor que $50 \mathrm{mg} \mathrm{L}^{-1}$. Assim, com base em estudos anteriores $[8,21,26,27]$ foi ajustado um modelo linear de estimativa da CSS a partir da banda do vermelho do sensor OLI (Banda 4). A Figura 4 apresenta o modelo empírico aplicados nas imagens de satélites e as estatísticas de validação.

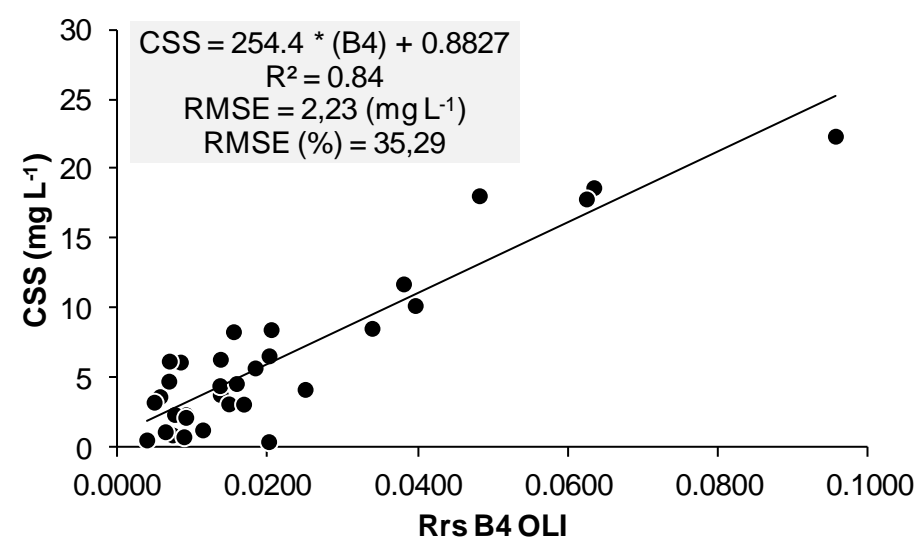

Figura 4. Modelo empírico preditivos da CSS a partir da Rrs simuladas para a banda do vermelho do sensor OLI/Landsat-8 (Rrs B4 OLI com fator de escala 0,0001).

Para a análise da dinâmica temporal e espacial do sedimento suspenso no Lago Catalão foi utilizada o acervo de dados de reflectância da superfície do satélite Landsat-8 (2013-2020) disponível pelo USGS. Os dados de reflectância de superfície nas imagens do OLI/Landsat-8 foram processados e extraídos através da plataforma Google Earth Engine [28]. 


\section{Resultados e Discussão}

$\mathrm{O}$ acervo de imagens do sensor OLI/Landsat-8 analisado neste estudo corresponde a um conjunto de 83 imagens adquiridas entre abril de 2013 e dezembro de 2020. A partir deste conjunto de dados foi possível estimar uma CSS média no Lago Catalão de $12,04 \mathrm{mg} \mathrm{L}^{-1}$ com máximo de $31,82 \mathrm{mg} \mathrm{L}^{-1}$ em abril de 2020 e mínimo de $1,62 \mathrm{mg} \mathrm{L}^{-}$ ${ }^{1}$ na imagem de abril de 2018. Entre os meses de dezembro e abril (enchente) a CSS média estimada foi de $8,20 \mathrm{mg} \mathrm{L}^{-1}(\mathrm{n}=28)$ enquanto na cheia, vazante e seca a CSS média foi de 15,09 $(n=23) ; 12,36(n=16)$ e 14,06 $(n=16) \mathrm{mg} \mathrm{L-1}$, respectivamente (Figura 5).

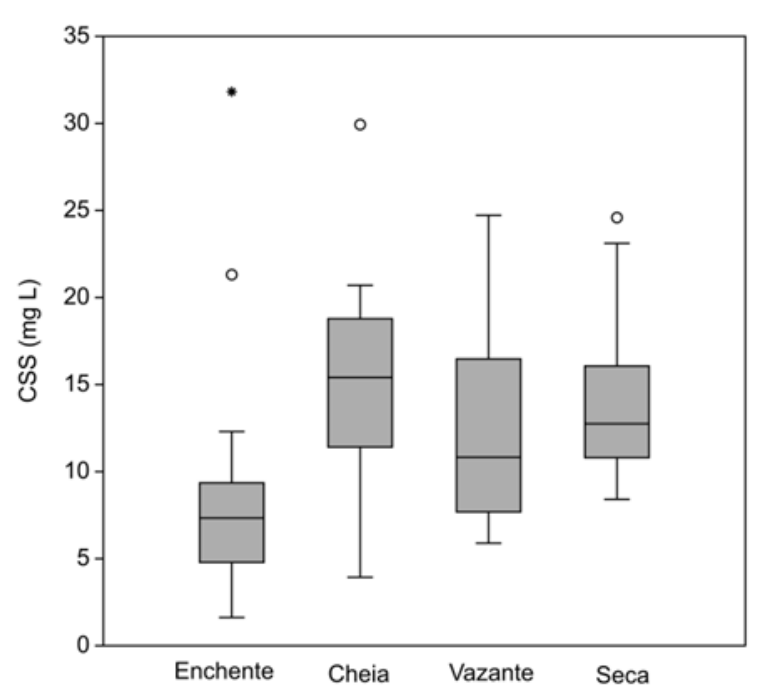

Figura 5. Concentração do sedimento suspenso (CSS) no Lago Catalão em diferentes períodos hidrológicos estimados com base nos dados OLI/Landsat-8.

O rio Solimões em Manacapuru $90 \mathrm{~km}$ a jusante da área de estudo possui CSS média anual da ordem de $133 \mathrm{mg} \mathrm{L}^{-1}$ [29], enquanto que o rio Negro próximo de sua foz apresenta uma CSS média anual inferior a $5 \mathrm{mg} \mathrm{L}^{-1}$ [30]. Neste estudo, a CSS média estimada para o Lago Catalão com as imagens Landsat-8 foi 2,5 vezes menor em comparação aos dados apresentados por Brito et al. [13] $\left(\sim 30,9 \mathrm{mg} \mathrm{L}^{-1}\right)$. No entanto, os dados destes autores foram coletados entre novembro de 2004 e agosto de 2005, ou seja, em um ano com seca extrema na bacia, o que pode ter contribuído para a maior concentração de sedimento suspenso observado. Assim, em baixa profundidade - lago está sujeito a ressuspensão do sedimento, que podem ser associadas às atividades antrópicas.

A concentração média anual de sedimentos suspensos do Lago Catalão (12,04 mg L $\left.{ }^{-1}\right)$ ficou próxima da estimativa realizada com dados MODIS no estudo de FassoniAndrade e Paiva [31], os quais classificou este lago como um ambiente de moderada concentração de sedimento suspenso na superfície d'água (com CSS variando anualmente de 10 a $35 \mathrm{mg} \mathrm{L}^{-1}$ ).

A série histórica da CSS estimada no período de abril de 2013 e dezembro de 2020 é apresentada na Figura 6. De forma geral os picos de CSS que ocorrem próximo do período de cheia são relacionados ao aporte de sedimentos suspensos oriundos do rio Solimões. Já os picos de CSS durante a vazante podem ser relacionados ao período que o Lago Catalão reduz área, a profundidade e o volume de água, o que pode contribuir para que o sedimento suspenso fique mais concentrado. 


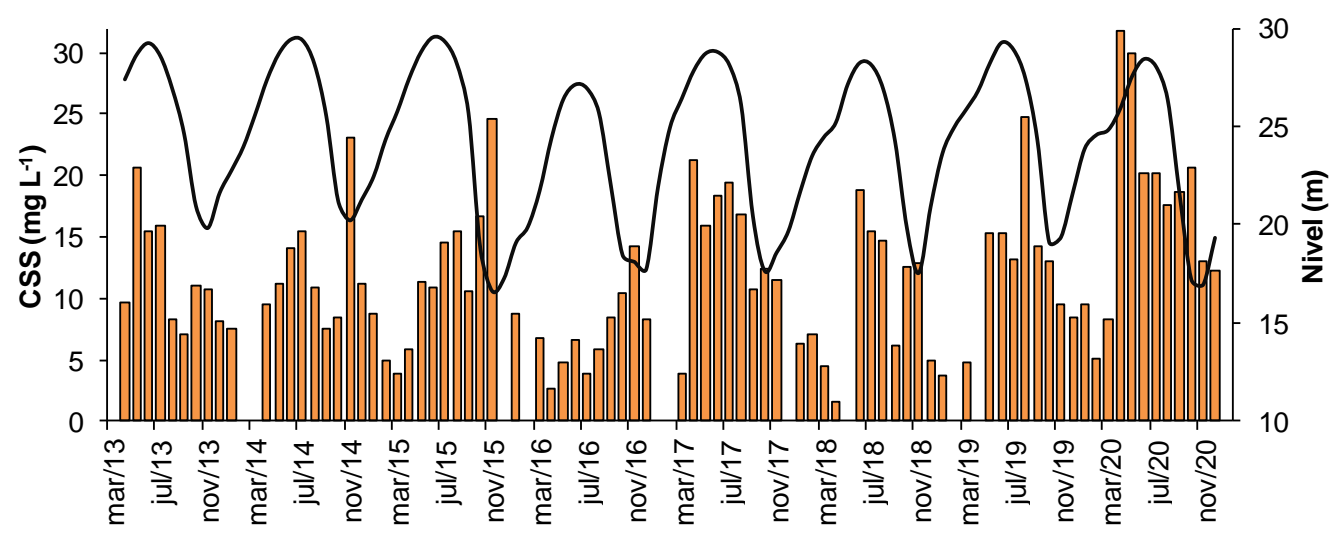

Figura 6. Série temporal da CSS derivadas a partir de imagens Landsat-8 (barras) no Lago Catalão e a variabilidade do nível do rio Negro em Manaus (linha).

Entre o período de transição da enchente (abril) e o final da cheia (julho) o fluxo de água do rio Solimões extravasa os canais que conectam os lagos e inunda toda a planície, em um processo que intensifica a circulação de água e nutrientes nos lagos, que por sua vez contribui para aumento da CSS. A Figura 7 mostra a dinâmica espaço-temporal do sedimento suspenso sobre a ilha Xiborena e no Lago Catalão entre maio de 2017 e março de 2018. O Lago Catalão recebe grande quantidade de águas de ambos os rios, sendo a influência do rio Negro maior no período de enchente em virtude da menor CSS mapeada na imagem OLI/Landsat-8 de março de 2018 (Figura 7c).
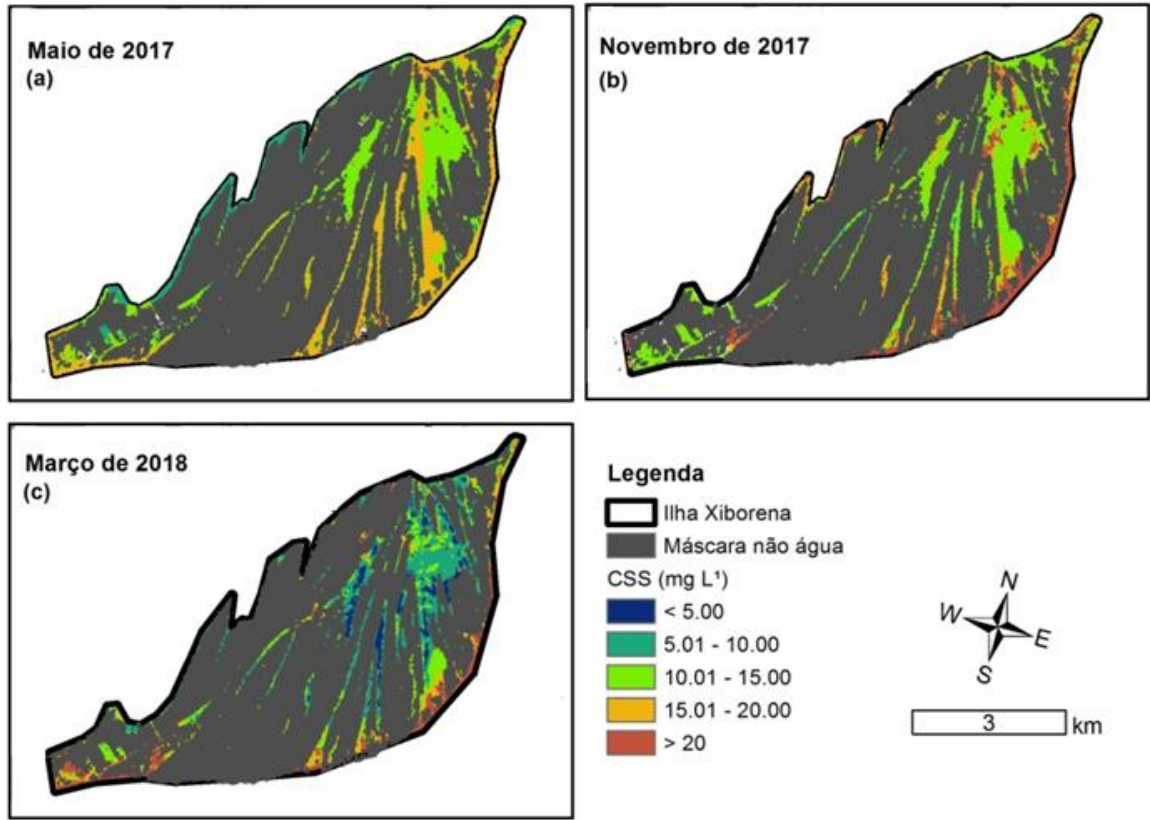

Figura 7. Distribuição espacial da CSS sobre a ilha Xiborena e o Lago Catalão durante a cheia (a) e seca (b) de 2017 e a enchente de 2018 (c).

A região central do Lago Catalão possui uma área inferior a $01 \mathrm{~km}^{2}$, tamanho que dificulta o uso de sensores orbitais de revisita diária e de baixa resolução espacial, como o MODIS (Satélites Terra e Aqua) e o OLCI (Sentinel-3). Com uma resolução espacial de 30 metros o sensor OLI/Landsat-8 consegue detectar tanto a área do Lago 
Catalão como os estreitos canais que o conecta aos rios Negro e Solimões. Por outro lado, com uma resolução temporal de 16 dias este sensor possui capacidade de coletar de 22 a 23 imagens por ano, que por sua vez, podem apresentar elevada cobertura de nuvens, principalmente entre os meses de dezembro e abril (período chuvoso). Estas limitações em relação às resoluções temporais e espaciais do OLI/Landsat-8 podem ser superadas com o uso combinado de sensores orbitais com bandas espectrais semelhantes, como por exemplo, as constelações de satélites Sentinel-2 e os CubeSats Planet, aumentando assim capacidade de monitoramento do local [32].

\section{Conclusão}

Este trabalho demonstrou através de um conjunto de imagens do sensor OLI/Landsat8 a dinâmica espaço-temporal do sedimento suspenso no Lago Catalão, um lago de águas mistas localizado na região de confluência entre os rios Negro e Solimões. $O$ sedimento suspenso no Lago Catalão apresenta um regime com menor concentração no período de enchente do rio Negro, comportamento semelhante aos observados nos lagos da planície do rio Solimões e Amazonas, onde o pico de entrada da carga suspensa ocorre próximo do pico de cheia do rio.

Dados de sensoriamento são importantes ferramentas para a análise e compressão de fenômenos geográficos em sistemas aquáticos localizados na bacia Amazônica, como a troca de sedimentos entre o rio e planície. Desta forma, os resultados apresentados neste estudo, em conjunto com trabalhos já realizados, podem servir na avaliação de como a população ribeirinha produz o espaço geográfico no Lago Catalão, bem como identificar impactos oriundos de eventos climáticos extremos, como as grandes secas de 2005 e 2010 e as cheias de 2009 e 2012.

Por fim, o uso de imagens do satélite Landsat- 8 contribuiu para entender como a paisagem deste lago muda ao longo de um ano hidrológico alterando sua geomorfologia e qualidade da água utilizada pela população ribeirinha. No entanto, mais estudos sobre este sistema aquático são necessários, principalmente com aplicações de sensores de maior resolução temporal e espacial, especialmente na aquisição de dados durante o período chuvoso, bem como na avaliação da influência da atmosfera e da reflexão especular da água (sunglint) sobre as imagens Landsat-8.

\section{Referências}

[1] Junk W., Bayley P., Sparks R. The Flood Pulse Concept in River-Floodplain Systems. Canadian Journal of Fisheries and Aquatic Sciences 1989; 106. Disponível em:

<https://www.waterboards.ca.gov/waterrights//water_issues/programs/bay_delta/docs/c mnt081712/sldmwa/junketal1989.pdf >. Acesso em: 09 de mar. 2021.

[2] Filizola N.P., Guyot J.L., Molinier M., Guimarães V. Caracterização hidrológica da Bacia Amazônica. In Rivas A., Freitas C.E. de C., (eds). Amazônia uma perspectiva interdisciplinar. EDUA: Manaus; 2002.

[3] Bittencourt M.M., Amadio S.A. Proposta para identificação rápida dos períodos hidrológicos em áreas de várzea do rio Solimões-Amazonas nas proximidades de Manaus. Acta Amazonica 2007; 37(2): 303-308. https://doi.org/10.1590/s004459672007000200019.

[4] Barbosa C.C.F., Novo E.M.L.M., Martins V.S. Introdução ao sensoriamento remoto de sistemas aquáticos: princípios e aplicações; 1a Edição. Instituto Nacional de 
Pesquisas Espaciais: São José dos Campos; 2019. Disponível em: <http://www.dpi.inpe.br/labisa/livro/>. Acesso em 10/03/2021.

[5] Gualtieri C., lanniruberto M., Filizola N., Santos R., Endreny T. Hydraulic complexity at a large river confluence in the Amazon basin. Ecohydrology 2017; 10(7): e1863. <https://doi.org/10.1002/eco.1863>.

[6] Laraque A., Guyot J.L., Filizola N. Mixing processes in the Amazon River at the confluences of the Negro and Solimões Rivers, Encontro das Águas, Manaus, Brazil. Hydrological Processes 2009; 23(22): 3131-3140. <https://doi.org/10.1002/hyp.7388>.

[7] Filizola N.P., Spínola N., Arruda W., Seyler F., Calmant S., Da Silva J. The Rio Negro and Rio Solimões confluence point - hydrometric observations during the 2006/2007 cycle. In River, Coastal and Estuarine Morphodynamics; Taylor \& Francis Group: Santa Fe, 2009; p. 1003-1006.

[8] Marinho T., Filizola N., Martinez J.M., Armijos E., Nascimento A. Suspended Sediment Variability at the Solimões and Negro Confluence between May 2013 and February 2014. Geosciences 2018; 265 . https://doi.org/10.3390/geosciences8070265.

[9] Moreira-Turcq P.F., Seyler P., Guyot J.L., Etcheber H. Characteristics of organic matter in the mixing zone of the Rio Negro and Rio Solimões of the Amazon River. Hydrological Processes 2003; 17(7): 1393-1404. https://doi.org/10.1002/hyp.1291.

[10] Sternberg H.O.R. A Água e o Homem na Várzea do Careiro; Museu Paraense Emílio Goeldi: Belém, 1998.

[11] Ramos M.F.L., Wachholz F., Silva Neto J.C.A. Qualidade dos recursos hídricos na comunidade flutuante lago do Catalão, Iranduba -AM. Caminhos de Geografia 2020; 21(73): 98-115. https://doi.org/10.14393/RCG217347290.

[12] Allard T., Ponthieu M., Weber T., Filizola N., Guyot J.L., Benedetti M. Nature and properties of suspended solids in the Amazon Basin. Bulletin de la Societe Geologique de France 2002; 173(1): 67-75. https://doi.org/10.2113/173.1.67.

[13] Brito J.G. de, Alves L.F., Espirito Santo H.M.V. Seasonal and spatial variations in limnological conditions of a floodplain lake (lake Catalão) connected to both the Solimões and Negro Rivers, Central Amazonia. Acta Amazonica 2014; 44(1): 121-133. http://dx.doi.org/10.1590/S0044-59672014000100012.

[14] Latrubesse E.M. Amazon lakes. In: Bengtsson L., Herschy R.W., Fairbridge R.W. (eds) Encyclopedia of Lakes and Reservoirs. Encyclopedia of Earth Sciences Series. Springer: Dordrecht; 2012. https://doi.org/10.1007/978-1-4020-4410-6_36.

[15] Novo E.M.L.M., Barbosa C., Freitas R.M. de. Sistemas Aquáticos Continentais. In: O sensor Modis e suas aplicações ambientais no Brasil; Rudorfff, B.F.T., Shimabukuro, Y.E., Ceballos, J.C., Eds.; Editora Parênteses: São José dos Campos, 2007; p. 265275.

[16] Pinheiro L., Cardoso A.C. A comunidade flutuante Lago Catalão-Iranduba AM: Um tecido urbano sobre as águas. Revista de Morfologia Urbana 2019; 7(2): e00114e00114. https://doi.org/10.47235/rmu.v7i2.114.

[17] Leite R.G., Silva J.V.V. da, Freitas C.E. Abundância e distribuição das larvas de 
peixes no Lago Catalão e no encontro dos rios Solimões e Negro, Amazonas, Brasil. Acta Amazonica 2006; 36(4): 557-562. https://doi.org/10.1590/S004459672006000400018.

[18] Röpke C.P., Amadio S.A., Winemiller K.O., Zuanon J. Seasonal dynamics of the fish assemblage in a floodplain lake at the confluence of the Negro and Amazon Rivers. Journal of Fish Biolology 2016; 89(1): 194-212. https://doi.org/10.1111/jfb.12791.

[19] Almeida F.F. de, Melo S. Considerações limnológicas sobre um lago da planície de inundação amazônica (lago Catalão - Estado do Amazonas, Brasil). Acta Scientiarum Biological Sciences 2009; 31(4): 387-395. https://doi.org/10.4025/actascibiolsci.v31i4.4641.

[20] Sioli H. The Amazon and its main affluents: Hydrography, morphology of the river courses, and river types. In: Sioli $\mathrm{H}$. (ed) Amazon Limnology and landscape ecology of mighty tropical river and its basins. Springer Netherlands: Dordrecht; 1984, p. 127-165. https://doi.org/10.1007/978-94-009-6542-3.

[21] Martinez J.M., Espinoza-Villar R., Armijos E., Silva Moreira L. The optical properties of river and floodplain waters in the Amazon River Basin: Implications for satellite-based measurements of suspended particulate matter. Journal of Geophysical Research: Earth Surface 2015; 120(7): $1274-1287$. https://doi.org/10.1002/2014JF003404.

[22] Lobo J.F.L. Aplicações do sensoriamento remoto em águas continentais: estudos de caso. In: Barbosa C.C.F., Novo E.M.L.M., Martins V.S. (eds) Introdução ao sensoriamento remoto de sistemas aquáticos princípios e aplicações. Instituto Nacional de Pesquisas Espaciais: São José dos Campos; 2019, p. 136-152.

[23] Messager M.L., Lehner B., Grill G., Nedeva I., Schmitt O. Estimating the volume and age of water stored in global lakes using a geo-statistical approach. Nature Communications 2016; 7: 13603. https://doi.org/10.1038/ncomms13603.

[24] Mobley C.D. Estimation of the remote-sensing reflectance from above-surface $\begin{array}{lllll}\text { measurements. } \quad \text { Applied } \quad \text { Optics 1999; 38(36): } & \text { 7442-7455. }\end{array}$ https://doi.org/10.1364/AO.38.007442.

[25] HYBAM. Observation Service SO HYBAM. Disponível em: <http://www.orehybam.org >. Acesso em 29/12/2018.

[26] Chelotti, G.B.; Martinez, J.M.; Roig, H.L.; Olivietti, D. Space-Temporal analysis of suspended sediment in low concentration reservoir by remote sensing. Brazilian Journal of Water Resources 2019; 24(17): 1-15. https://doi.org/10.1590/23180331.241920180061.

[27] Marinho R.R. Integração de dados de campo e sensoriamento remoto no estudo do fluxo de água e matéria no Arquipélago de Anavilhanas, Rio Negro - Amazonas, Brasil. Tese de Doutorado. Manaus: Instituto Nacional de Pesquisas da Amazônia; 2019.

[28] USGS Landsat 8 Surface Reflectance Tier 1. Disonível em: $<$ https://developers.google.com/earthengine/datasets/catalog/LANDSAT_LC08_C01_T1_SR>. Acesso em 04 de jan. 2021. 
[29] Espinoza-Villar R., Martinez J.-M., Armijos E., Espinoza J.-C., Filizola N., Dos Santos A., Willems B., Fraizy P., Santini W., Vauchel P. Spatio-temporal monitoring of suspended sediments in the Solimões River (2000-2014). Comptes Rendus Geoscience 2018; 350(1-2): 4-12. https://doi.org/10.1016/j.crte.2017.05.001.

[30] Marinho R.R., Filizola Junior N.P., Cremon É.H. Analysis of Suspended Sediment in the Anavilhanas Archipelago, Rio Negro, Amazon Basin. Water 2020; 12(4): 1073. https://doi.org/10.3390/w12041073.

[31] Fassoni-Andrade A.C., Paiva R.C.D. de. Mapping spatial-temporal sediment dynamics of river-floodplains in the Amazon. Remote Sensing of Environment 2019; 221: 94-107. https://doi.org/10.1016/j.rse.2018.10.038.

[32] Maciel D., Novo E., Carvalho L.S. de, Barbosa C., Júnior R.F., Lobo F. de L. Retrieving Total and Inorganic Suspended Sediments in Amazon Floodplain Lakes: A Multisensor Approach. Remote Sensing. 2019, 11(15): 1744. https://doi.org/10.3390/rs11151744.

\section{Agradecimentos}

Agradecemos o apoio da Universidade Federal do Amazonas (UFAM) pelo apoio ao projeto de iniciação cientifica (PIB-E/0055/2020). Este trabalho é uma contribuição ao projeto "BASNE - Balanço de Água e Sedimentos no baixo curso do Rio Negro" desenvolvido por pesquisadores do grupo de Pesquisa Hidrossistemas e o Homem na Amazônia (H2A/UFAM) com apoio da Fundação de Amparo a Pesquisas do Estado do Amazonas (FAPEAM).

\section{Autores}

Rogério Ribeiro Marinho ${ }^{1, \star}$, Flávio Wachholz², Renata Souza ${ }^{1}$

1. Departamento de Geografia, Universidade Federal do Amazonas, Ave. General Rodrigo Otávio Jordão Ramos 6200, Campus Universitário, Coroado I, Manaus CEP 69077-000, Brasil.

2. Escola Normal Superior, Universidade do Estado do Amazonas, Avenida Djalma Batista 2470, Chapada 69050-010 - Manaus, AM - Brasil.

* Autor para correspondência: rogeo@ufam.edu.br 